\title{
Driving Behavior Analysis for Smartphone-based Insurance Telematics
}

\author{
Johan Wahlström, Isaac Skog, and Peter Händel \\ ACCESS Linnaeus Center, Dept. of Signal Processing \\ KTH Royal Institute of Technology \\ Stockholm, Sweden \\ \{jwahlst, skog, ph\}@kth.se
}

\begin{abstract}
Insurance telematics programs are continuously gaining market shares in the automotive insurance industry. By recording data on drivers' behavior, the information asymmetry between the policyholder and the insurer is reduced, enabling a granular risk differentiation based on the true risk levels of the drivers. However, the growth of the insurance telematics industry is being held up by large logistic costs associated with the process of acquiring data. As a result, several market participants have started looking towards smartphone-based solutions, which have the potential of easing and improving the data collection process for both policyholders and insurers.

In this paper, we present a unified framework highlighting the challenges of smartphone-based driver behavior analysis. Since all driver behavior analysis relies on access to accurate navigation data, we first address the intermediate step of smartphone-based automotive navigation. The considered topics include estimation of the smartphone's orientation with respect to the vehicle, classification of the smartphoneowner as a passenger or driver, and navigation in GNSSchallenged areas. Once a driver-specific high-performance navigation solution has been obtained, it can be used to extract information on the driver's behavior. We review the most commonly employed driving events, and discuss some of the difficulties inherent in detecting these events.
\end{abstract}

\section{Categories and Subject Descriptors}

H.4.m [Information Systems Applications]: Miscellaneous

\section{Keywords}

Smartphones; Insurance Telematics; Driver Behavior Analysis.

\section{INTRODUCTION}

Traditionally, the automotive insurance industry has relied on measures such as the drivers' gender and age, place of residence, and car model, to set the premiums offered

Permission to make digital or hard copies of part or all of this work for personal or classroom use is granted without fee provided that copies are not made or distributed for profit or commercial advantage, and that copies bear this notice and the full citation on the first page. Copyrights for third-party components of this work must be honored. For all other uses, contact the owner/author(s). Copyright is held by the author/owner(s).

WPA'15, May 22, 2015, Florence, Italy.

ACM 978-1-4503-3498-3/15/05.

http://dx.doi.org/10.1145/2753497.2753535 .

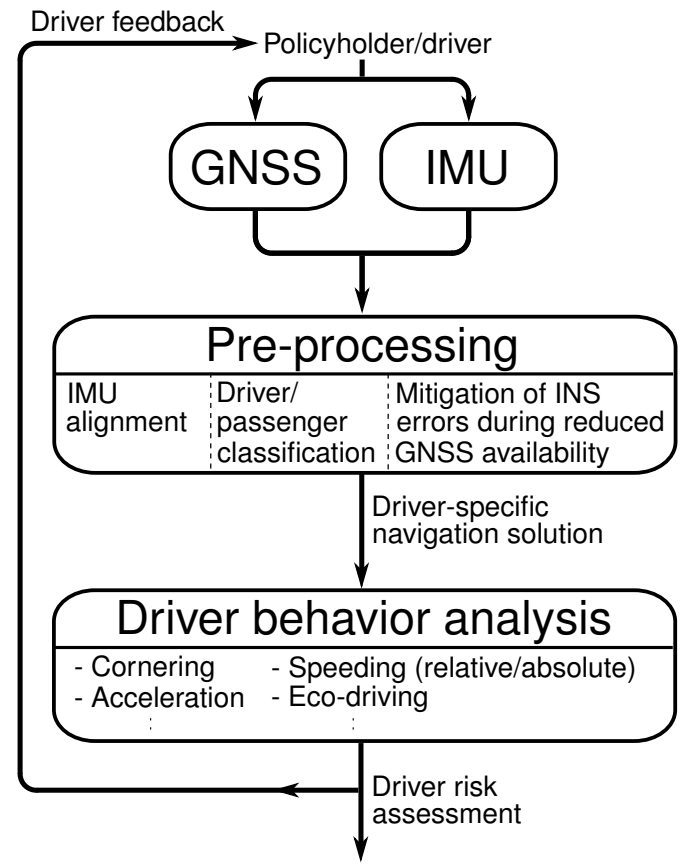

Figure 1: Flow diagram illustrating the process in going from sensor measurements to a driver risk assessment in smartphone-based insurance telematics.

to each driver. However, these criteria will provide a fairly low predictability of the risks taken by a driver while on the road. As a consequence, the interest in insurance telematics, i.e., usage-based automotive insurance where data on driving behavior is collected by means of telecommunication, has grown at a rapid pace during the last years. The total subscriber base is expected to reach 85.5 million by 2018 [1]. As of yet, the largest market participants have collected data by the use of in-vehicle sensors accessed through the vehicle's on-board diagnostics port. At this point, however, several insurers and telematics service providers have started looking towards smartphone-based solutions [2]. Thanks to the large number of embedded sensors, smartphones are able to provide insurers with rich driving data (the typical process is illustrated in Fig. 1). Collecting data by the use of smartphones is also generally cheaper and less logistically demanding than employing vehicle-fixed sensors. In addition, smartphones have proven to be excellent platforms for providing the user with driving feedback and allowing for advanced user interaction [3]. This is expected to increase 
both overall driver safety and the transparency of automotive insurance programs.

The two primary information sources in smartphone-based insurance telematics are the global navigation satellite system (GNSS) receiver and the inertial measurement unit (IMU). A cellular network assisted GNSS receiver will typically provide measurements of position, speed, and course within seconds after startup. The most prevalent error sources stem from ionospheric delays, clock errors, and multipath signal propagation [4]. The IMU provides measurements of specific force and angular velocity, obtained from a three-axis accelerometer and a three-axis gyroscope, respectively. Measurements provided by the IMU are subject to both a timevarying bias and random noise. GNSS and IMU measurements are often fused in a GNSS-aided inertial navigation system (INS) [5]. The employed navigation filter will integrate the IMU measurements in a prediction step, and then use GNSS measurements to bound the resulting navigation errors. GNSS-aided INSs will typically provide estimates of position, velocity, and attitude, and in addition also track the IMU bias. A smartphone-based GNSS-aided INS has previously been presented in [24]. Additional smartphoneembedded sensors include cameras, magnetometers, and microphones. Although all of these sensors have been used in isolated academic studies, their use in commercial telematics applications have been very limited. Vision-aided navigation algorithms are generally cumbersome in practical applications, due to their strict requirements on the smartphone's placement within the vehicle. Magnetometers are often redundant for navigation when GNSS and IMU measurements are available, and can be expected to be affected by magnetic disturbances from the vehicle engine [6]. Microphones obviously have limited abilities to collect data on driving behavior, but have previously been used for honk detection when constructing predictors of traffic intensity in developing countries [7].

In this paper, we present a unified framework highlighting the challenges of smartphone-based driver behavior analysis. The process in going from sensor measurements to a driver risk assessment is illustrated by Fig. 1. As all driving behavior analysis requires accurate navigation data, we first discuss low-level data processing designed to provide a high-performance navigation solution. The discussion is divided into three sections (see Sections 2-4) covering the following topics: IMU alignment, i.e., the problem of finding the relative orientation of the smartphone and the vehicle; driver/passenger classification, i.e., the problem of classifying the smartphone owner as a passenger or driver based on the position of the smartphone in the vehicle; and navigation in areas with reduced GNSS coverage or high levels of multipath propagation. Section 5 then reviews the most commonly employed driving events and illustrates how these can be used to assess the risk level of the driver. The paper is concluded in Section 6. For discussions on driver scoring (i.e., constructing a comparative risk assessment based on the driver behavior analysis) and driver feedback, we refer to $[3],[8]$, and references therein.

\section{IMU ALIGNMENT}

Several studies have investigated the problem of estimating the orientation of a vehicle-fixed smartphone with respect to the vehicle. The importance of this problem derives from the fact that measurements from smartphone-
Table 1: Standard methods for estimating the Euler angles (relative a tangent coordinate frame) required for smartphone-to-vehicle alignment.

\begin{tabular}{lll}
\hline \hline Object & Angle & Estimation method \\
\hline Vehicle & $\begin{array}{l}\text { Roll } \\
\text { Pitch }\end{array}$ & $\begin{array}{l}\text { Assume the vehicle is on a } \\
\text { flat surface i.e., approximate } \\
\text { both angles by zero. }\end{array}$ \\
\cline { 2 - 3 } & Yaw & $\begin{array}{l}\text { Estimate the direction of } \\
\text { the vehicle velocity from } \\
\text { GNSS measurements. } \dagger\end{array}$ \\
\hline Smartphone & Roll & $\begin{array}{l}\text { Estimate the gravity vector } \\
\text { from accelerometer } \\
\text { measurements. }\end{array}$ \\
\cline { 2 - 3 } & Pitch & $\begin{array}{l}\text { Estimate the direction of } \\
\text { the magnetic north from } \\
\text { magnetometer measurements. } \dagger\end{array}$ \\
\hline \hline
\end{tabular}

$\dagger$ The relative smartphone-to-vehicle orientation can also be directly estimated by studying the effect that vehicle

accelerations have on the accelerometer measurements.

embedded IMUs will be dependent on the orientation of the device. Hence, to be able to take advantage of smartphoneembedded IMUs on the same terms as dedicated vehiclefixed IMUs, one must first estimate the smartphone-to-vehicle orientation.

The estimation process can be described as separate estimations of the smartphone's and the vehicle's orientations with respect to a tangent frame. The vehicle is assumed to be on horizontal ground, i.e., both its roll and pitch angles are approximated by zero. The roll and pitch angles of the smartphone can be estimated by identifying the gravity vector from accelerometer measurements [5]. Following this, the smartphone's yaw vector is estimated using magnetometer measurements [9], while the vehicle's yaw angle is estimated using GNSS measurements. Another possibility is to directly estimate the relative yaw angle of the smartphone and the vehicle by studying the effect that accelerations in the forward direction of the vehicle frame have on the accelerometer measurements [10]. The standard methods are summarized in Tab. 1. As noted in [11], the assumption on the vehicle's pitch angle (approximating it by zero) can be relaxed by matching the smartphone's three dimensional velocity with the forward direction of the vehicle frame.

In practice, it is unrealistic to assume that the smartphone is constantly fixed with respect the vehicle. The change in position and velocity resulting from a user picking up the smartphone can most often be neglected when estimating the vehicle dynamics. However, changes in the smartphone's orientation with respect to the vehicle are more tricky to deal with if one seeks to utilize measurements from the IMU. Since the angular velocity (of the smartphone) resulting from a user picking up the smartphone is generally much larger than any angular velocity caused by vehicle maneuvers (see Fig. 2), having a non-stationary smartphone-tovehicle orientation will often prevent standard inertial navigation of the vehicle. In addition, the rotations can be expected to excite new error terms in the IMU, which were previously negligible, e.g., scale factor errors. Estimating a non-stationary smartphone-to-vehicle orientation is difficult in practice, and hence, the chosen method for IMU alignment typically must be restarted when the smartphone is once again fixed inside the vehicle. 


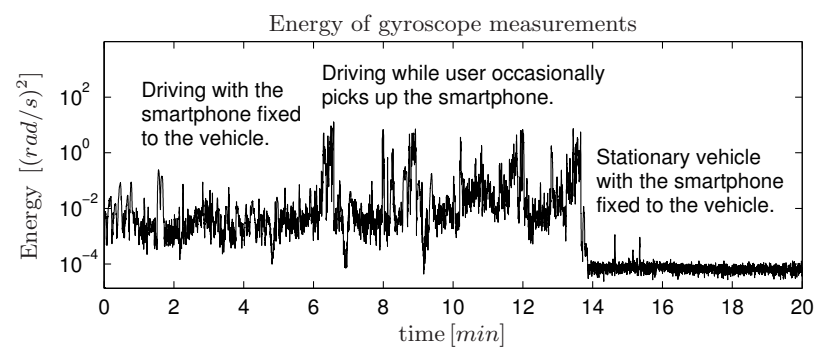

Figure 2: The energy of the gyroscope measurements as dependent on the smartphone dynamics (with respect to the vehicle) and the vehicle dynamics. The energy was averaged over a time window of $0.5[s]$.

\section{DRIVER/PASSENGER CLASSIFICATION}

Even though data has been recorded, its relevance for insurance purposes will depend on who the driver was and which vehicle was driven. This is especially important for smartphone apps that automatically start recording as soon as automotive driving is detected. The most easily implemented methods for driver/passenger classification make the assumption that each smartphone is placed close to its owner, and then attempts to estimate the smartphone's position in the vehicle using the collected navigation data. If the estimated smartphone position is near the driver's seat, it is concluded that the driver is the owner of the smartphone. These methods lead to low logistic costs and guarantee backwards compatibility with older vehicle models [12].

One option is to use the human motion dynamics of the driver or passenger to identify his or her seat within the vehicle [13]. However, this approach will obviously be sensitive to motion variations among individuals. Alternatively, the position of the smartphone can be determined by exploiting differences in the collected data of vehicle dynamics, resulting from the placement of the smartphone. In these cases, one must assume that data is available from either two smartphones, or from one smartphone and one reference system. Due to the time-correlated errors in the GNSS measurements, it is typically difficult to estimate the relative position of two smartphones directly from their GNSS position measurements, or from the position estimates provided by their associated GNSS-aided INSs. This is illustrated in Fig. 3, which shows the estimated relative position (in one horizontal dimension of the tangent frame) between two smartphones placed next to each other as a function of time. The estimates are computed as the mean of the difference in the estimated positions (provided by separate GNSS-aided INSs), up to the current period of time. Since the error in the estimated relative position is in the same order as the expected true difference in position between two smartphones, this will not be a reliable method for driver/passenger classification. Higher accuracy can be obtained by comparing the IMU measurements of the two smartphones. The difference in placement will be particularly evident when the vehicle corners (for identifying the lateral placement in the vehicle) [14] or when crossing a pothole or speed bump (for identifying the longitudinal placement in the vehicle) [15].

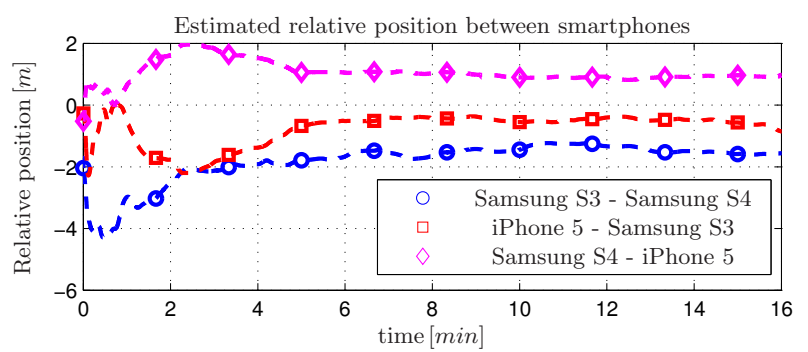

Figure 3: The estimated relative position between smartphones using their individually estimated positions. The true separation of the smartphones was in the order of $15[\mathrm{~cm}]$.

\section{GNSS-CHALLENGED AREAS}

Reduced GNSS availability or poor GNSS accuracy will typically degrade the navigation solution. However, even if the GNSS coverage is very poor, there are several ways to mitigate or reduce the error growth of INSs and extract valuable navigation data. In this section, four methods are discussed: map-matching, road-feature-aided navigation, nonholonomic constraints, and smoothing. It may be noted that all these methods can also be employed when GNSS is available. However, in these cases, the resulting improvement in estimation accuracy will be modest at best.

In map-matching, maps of road networks are used to improve the position estimates, or to extract information on the vehicle's path [16]. The map typically represents the road network as a mathematical graph composed of arcs and nodes. The accuracy of the map-matching will depend on e.g., the accuracy of the map, the accuracy and update rate of the a priori position estimates, the employed mapmatching technique, and the road density. As noted in [17], the primary challenge of smartphone-based map-matching lies in managing the low performance of built-in GNSS receivers. In addition, the GNSS receivers will in many cases be duty-cycled to preserve energy. To cope with the low update rate resulting from this, probabilistic map-matching will often be necessary. This means that the map-matching algorithm stores several potential true paths with their corresponding probabilities. One of the shortcomings of mapmatching is that the requirements on the a priori position estimates are higher in urban areas where the road density is high [18]. Unfortunately, this is also where tall buildings are most likely to block satellite signals and where GNSS multipath propagation is the most severe.

Road-feature-aided navigation resembles map-matching in the sense that road maps are used to improve the position estimate. However, the matching process is not primarily based on a priori position estimates, but on the identification of road-features. A road-feature identified using the smartphone sensors can often be associated with several similar road-features on the map, and hence, road-feature-aided navigation will typically require sophisticated data association algorithms. In what follows, we discuss the most commonly employed road-features.

The study in [19] presented a smartphone-based INS aided by speed bumps. All speed bumps were detected using accelerometer measurements in the vertical axis of the vehicle frame. The data association was based on a hidden Markov model with transition probabilities derived from the connec- 
tivity of the bumps. Field experiments in parking garages illustrated the feasibility of the method. Previously studied road-features have also included: vehicle stops at traffic lights, with a probability distribution of the queue length that varies with the time of day; vehicle turns, detected using magnetometers or integrated gyroscope measurements; and significant road slopes, detected using patterns in the estimated vehicle pitch angle [20].

Obtaining accurate speed estimates from map-matched or road-feature-aided position estimates is generally difficult. This is a consequence of the well-known noise amplifying characteristic of the differentiation operator. However, the vehicle's speed can often be directly estimated despite poor GNSS coverage [21]. One way to do this is to approximate the vehicle trajectory with a circular motion during vehicle turns. In this case, estimates of the vehicle's speed can be readily derived from the angular velocity and acceleration measured by the IMU. The drift of the vehicle's speed can also be bounded by the detection of vehicle stops, so called zero-velocity-updates. At last, we note that all speed bumps or potholes typically will be hit by both the vehicle's front wheels and its rear wheels [22]. Each bump will then result in two peaks in the IMU measurements, and hence, the vehicle's speed can be inferred using knowledge of the vehicle's wheelbase.

In practice, detectable road-features will often be far apart, and hence, road-feature-aided navigation should be complemented by standard map-matching algorithms to avoid divergence of the navigation filter. Moreover, the positions of road-features will in general not be known a priori. The problem of building a map of traffic lights and stop signs using only GNSS data has previously been considered in [23].

Several studies have proposed the use of non-holonomic constraints (NHCs) to reduce the error growth of the INS during GNSS outages [24]. The NHCs utilize that the velocity of the vehicle is roughly aligned with the forward direction of the vehicle frame. As a result, the number of degrees of freedom in the estimated velocity and orientation decreases from six (three-dimensional velocity and threedimensional orientation) to four (speed and three-dimensional orientation). Typically, the constraints are implemented as noisy measurements of zero velocity in the lateral and vertical directions of the vehicle frame. In contrast to map-based algorithms, NHCs can be used to improve the speed estimates even when the vehicle's initial position is unknown. However, NHCs will always require some knowledge or estimate of the smartphone-to-vehicle orientation.

Smoothing is a method of estimation where the value of a stochastic process at a specified time is estimated using measurements collected up until a later time point. In other words, smoothing is a non-casual estimation procedure which exploits future measurements to improve upon the estimation accuracy obtained in standard online filtering algorithms. Practical implementations of smoothing for vehicle navigation will typically be based on fixed-lag smoothers which utilize one additional GNSS measurement as compared to the standard filter [25]. With smoothing, the estimation uncertainty will be the largest in between GNSS measurements, and not right before the GNSS measurements as in the standard filter. It can be noted that smoothing algorithms by its very nature will always produce delayed solutions. Moreover, smoothing requires intermittent measurement updates, and is therefore not a feasible alter- a)

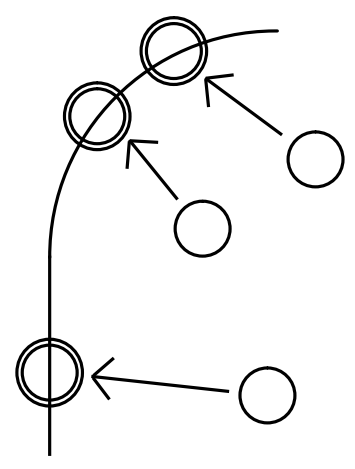

A priori position estimates.

Map-matched nodes.

c)

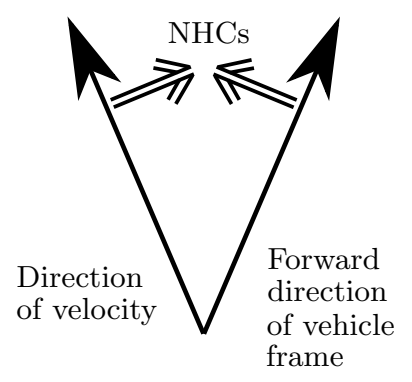

b)

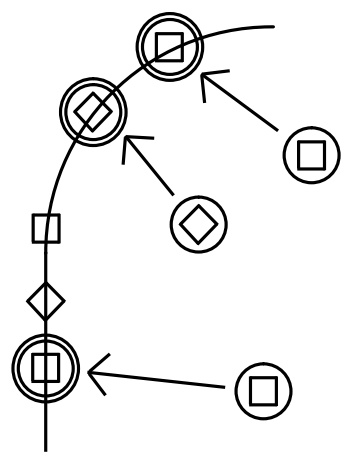

Road-feature 2.

d)

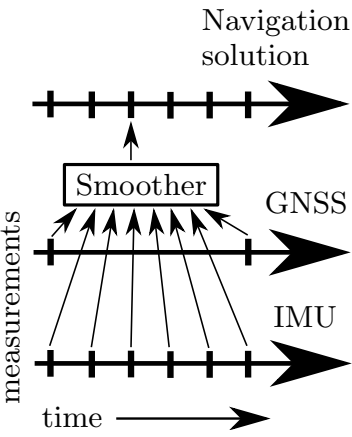

Road-feature 1.

Figure 4: Four methods for mitigation of INS errors during degraded GNSS availability: a) mapmatching; b) road-feature-aided navigation; c) nonholonomic constraints; d) smoothing. For simplicity, the map is illustrated as a continuous twodimensional curve.

native when neither map information nor GNSS signals are available. Fig. 4 illustrates the four described methods for mitigation of INS errors during degraded GNSS availability.

At last, we note that several of the frameworks presented in the literature for e.g., driver risk assessment, only make use of IMU measurements (see e.g., [26]). However, excluding GNSS measurements will generally reduce the number of observable modes in the IMU bias, and also make it harder to estimate the smartphone-to-vehicle orientation. This will then increase the logistic requirements on, e.g., sensor calibration and the placement of the smartphone in the vehicle.

\section{FIGURE OF MERITS AND DRIVING EVENTS}

When a sufficiently accurate navigation solution has been obtained, the provided data is used to analyze the driver's behavior. The analysis will result in a driver risk assessment, which then forms the basis of insurance premium adjustments [27]. A number of different figure of merits (FoMs) or driving events have been studied [3]. Several are based on the location of the vehicle at a specified time, and have 
low requirements on accuracy and resolution. These include the driver's location during trips, the elapsed distance of the trips, and the time of day when the trip was made. All of these FoMs are easily observable, given that driving data exists. More intricate FoMs are often based on sudden driving maneuvers, spanning very short periods of time. We now proceed with a more detailed discussion on a number of these FoMs: cornering, harsh acceleration and braking, and speeding. All the FoMs discussed in this section have been used by commercial telematics providers (see e.g., [28]). The section concludes with some notes on actuarial relevance and data privacy.

Risk assessment of cornering events is often made by estimating the forces exerted on the vehicle. Appropriate test statistics can be derived based on the dynamics of skidding and rollovers [29]. Choosing the threshold for detection of dangerous events determines the tradeoff between making accurate detections (easier at lower thresholds) and making relevant detections (easier at higher thresholds).

Harsh acceleration or braking has been used as an indicator of unsafe driving in several insurance programs. The estimated acceleration is generally obtained directly from the accelerometer measurements [30]. High frequency noise can often be mitigated by exploiting that the bandwidth of the longitudinal acceleration in most cases is less than $2[\mathrm{~Hz}]$ [4]. Similarly to detecting dangerous cornering, there is an inherent tradeoff between detection accuracy and actuarial relevance in choosing the detection thresholds. To illustrate the importance of utilizing IMU measurements for detection of short-time events, we studied detection of harsh (longitudinal) acceleration and braking using three smartphones: one Samsung S3, one Samsung S4, and one iPhone 5. We simultaneously collected reference data using a Microstrain 3DM-GX3-35. All of the smartphones were fixed to the vehicle during the entire trip, and their IMUs were sampled at $10[\mathrm{~Hz}]$. The studied data set consisted of approximately 30 minutes of data, and used a detection threshold of $1.5\left[\mathrm{~m} / \mathrm{s}^{2}\right]$. Tab. 2 shows the time-averaged rootmean-square errors (RMSEs) of the acceleration estimates provided by the smartphones in two cases: when estimating the vehicle's acceleration from first-order difference quotients constructed from the GNSS measurements of speed and time; and when estimating the vehicle's acceleration using the estimates provided by a GNSS-aided INS. The latter estimates were low-pass filtered with a cutoff frequency of $2[H z]$. As can be seen, employing a GNSS-aided INS not only increases the estimation rate, but also reduces the RMSEs with almost 50\%. Seven instances of harsh acceleration or braking were identified from the reference data. We defined all instances which were missed (by a margin of at least 2 seconds) by the smartphones as missed detections, and all false detections provided by the smartphones as false alarms. The number of missed detections and false alarms provided by the smartphones are given in Tab. 3 and Tab. 4 . As seen from the figures, the GNSS-aided INS clearly outperforms the estimator only based on GNSS measurements.

Speeding can be measured in absolute terms, in comparison to the normal or prevalent traffic flow, or in comparison to the legal speed limit [31]. The estimation accuracy of absolute speeding is obviously only limited by the accuracy of the vehicle's estimated speed. Comparing the vehicle speed to the prevailing traffic flow requires a high penetration rate of measurement probes, or other means of collecting infor-
Table 2: Time-averaged root-mean-square errors of acceleration estimates (given in $\left[\mathrm{m} / \mathrm{s}^{2}\right]$ ).

\begin{tabular}{l|lll}
\hline \hline & Samsung S3 & Samsung S4 & iPhone 5 \\
\hline GNSS & 0.43 & 0.43 & 0.35 \\
GNSS-aided INS & 0.26 & 0.25 & 0.21
\end{tabular}

Table 3: Detection accuracy of harsh braking using GNSS measurements. There were 7 true events.

\begin{tabular}{l|lll}
\hline \hline & Samsung S3 & Samsung S4 & iPhone 5 \\
\hline Missed detections & 3 & 5 & 2 \\
False alarms & 4 & 3 & 2
\end{tabular}

Table 4: Detection accuracy of harsh braking using GNSS-aided INSs. There were 7 true events.

\begin{tabular}{l|lll}
\hline \hline & Samsung S3 & Samsung S4 & iPhone 5 \\
\hline Missed detections & 0 & 0 & 1 \\
False alarms & 0 & 1 & 0
\end{tabular}

mation regarding the traffic in general. Detections of speed limit violations are only possible if the vehicle is first accurately mapped to a road-segment.

The actuarial relevance of a FoM depends on its correlation with insurance claims. A rigorous correlation analysis requires access to both historical insurance claims and associated driving data, something which few market participants have today. Both policyholders and authorities have raised concerns regarding the privacy intrusion of telematics services [32]. To mitigate privacy concerns, driving scores may be calculated directly in the smartphone, and hence, sensitive sensor data can be deleted without being sent to a central server.

\section{CONCLUSION}

Motivated by the growing interest in smartphone-based insurance telematics, this paper has highlighted some of the main challenges of smartphone-based driver behavior analysis. Three major obstacles in the construction of a driverspecific navigation solution were identified: the unknown orientation of the smartphone with respect to the vehicle; the unknown identity of the driver; and the problem of mitigating INS errors during prolonged GNSS outages. We reviewed state-of-the-art solutions and demonstrated typical characteristics of collected data by several numerical examples. The paper concluded with a discussion on figure-ofmerits and driving events that are commonly used as input in the process of adjusting insurance premiums.

\section{REFERENCES}

[1] "Insight report: Technology in action - A roadmap for insurance telematics," Oct. 2014, ReportsnReports.

[2] P. Olson, "Insurers aim to track drivers through smartphones," May 2014, Forbes.

[3] P. Händel, I. Skog, J. Wahlström, F. Bonawiede, R. Welch, J. Ohlsson, and M. Ohlsson, "Insurance telematics: Opportunities and challenges with the smartphone solution," IEEE Intell. Transport. Syst. Mag., vol. 6, no. 4, pp. 57-70, Oct. 2014.

[4] I. Skog and P. Händel, "In-car positioning and navigation technologies - A survey," IEEE Trans. 
Intell. Transport. Syst., vol. 10, no. 1, pp. 4-21, March 2009.

[5] P. D. Groves, Principles of GNSS, inertial, and multisensor integrated navigation systems, 1 st ed. Artech House, 2008.

[6] S. Godha, M. G. Petovello, and G. Lachapelle, "Performance analysis of MEMS IMU/HSGPS/magnetic sensor integrated system in urban canyons," in Position, Location and Navigation Group, Long Beach, CA, Sep. 2005.

[7] P. Mohan, V. N. Padmanabhan, and R. Ramjee, "Nericell: Rich monitoring of road and traffic conditions using mobile smartphones," in Proc. 6th ACM Conf. Embedded Netw. Sensor Syst., Raleigh, NC, 2008, pp. 323-336.

[8] P. Händel, I. Skog, M. Ohlsson, and J. Ohlsson, "Smartphone instrumentation for insurance telematics," in Proc. IEEE Int. Conf. Instrum. Meas. Technol., Pisa, Italy, May 2015.

[9] N. Promwongsa, P. Chaisatsilp, S. Supakwong, C. Saiprasert, T. Pholprasit, and P. Prathombutr, "Automatic accelerometer reorientation for driving event detection using smartphone," in 13th ITS Asia Pacific Forum, Auckland, New Zealand, Apr. 2014.

[10] J. Paefgen, F. Kehr, Y. Zhai, and F. Michahelles, "Driving behavior analysis with smartphones: insights from a controlled field study," in Proc. 11th Conf. Mobile and Ubiquitous Multimedia, Luleå, Sweden, Dec. 2012, pp. 36:1-8.

[11] J. Wahlström, I. Skog, and P. Händel, "IMU alignment for smartphone-based automotive navigation," in Proc. 18th IEEE Int. Conf. Inf. Fusion, Washington, DC, 2015.

[12] H. Chu, V. Raman, J. Shen, A. Kansal, V. Bahl, and R. R. Choudhury, "I am a smartphone and I know my user is driving," in Proc. 6th Int. Conf. Commun. Syst. Netw. Bangalore, India: Other, Jan. 2014.

[13] C. Bo, X. Jian, and X. Li, "TEXIVE: detecting drivers using personal smart phones by leveraging inertial sensors," CoRR, vol. abs/1307.1756, 2013.

[14] Y. Wang, J. Yang, H. Liu, Y. Chen, M. Gruteser, and R. P. Martin, "Sensing vehicle dynamics for determining driver phone use," in Proc. 11th Annu. Int. Conf. Mobile Systems, Appl., Services, Taipei, Taiwan, Jun. 2013, pp. 41-54.

[15] Z. He, J. Cao, X. Liu, and S. Tang, "Who sits where? Infrastructure-free in-vehicle cooperative positioning via smartphones," Sensors, vol. 14, no. 7, pp. 11605-11628, Jun. 2014.

[16] M. A. Quddus, W. Y. Ochieng, and R. B. Noland, "Current map-matching algorithms for transport applications: State-of-the art and future research directions," Transport. Research Part C: Emerg Technol., vol. 15, no. 5, pp. 312 - 328, 2007.

[17] M. Bierlaire, J. Chen, and J. Newman, "Modeling route choice behavior from smartphone GPS data," Ecole Polytechnique Fédérale de Lausanne, Tech. Rep., 2010.

[18] M. Alrefaie, I. Carreras, F. Cartolano, R. Di Cello, and F. De Rango, "Map matching accuracy: Energy efficient location sampling using smartphones," in Proc. 16th Int. IEEE Conf. Intell. Transport. Syst.,
The Hague, the Netherlands, Oct. 2013, pp. 2243-2248.

[19] G. Tan, M. Lu, F. Jiang, K. Chen, X. Huang, and J. Wu, "Bumping: A bump-aided inertial navigation method for indoor vehicles using smartphones," IEEE Trans. Parallel Distrib. Syst., vol. 25, no. 7, pp. 1670-1680, Jul. 2014.

[20] C. Bo, X.-Y. Li, T. Jung, and X. Mao, "Smartloc: Sensing landmarks silently for smartphone based metropolitan localization," CoRR, vol. arXiv:1310.8187, 2013.

[21] H. Han, J. Yu, H. Zhu, Y. Chen, J. Yang, Y. Zhu, G. Xue, and M. Li, "Senspeed: Sensing driving conditions to estimate vehicle speed in urban environments." in Conf. Comput. Commun., Toronto, Canada, Apr. 2014, pp. 727-735.

[22] J. Eriksson, L. Girod, B. Hull, R. Newton, S. Madden, and H. Balakrishnan, "The pothole patrol: Using a mobile sensor network for road surface monitoring," in 6th Annu. Int. Conf. Mobile Syst. Appl. Services, Breckenridge, CO, Jun. 2008.

[23] S. Hu, L. Su, H. Liu, H. Wang, and T. F. Abdelzaher, "Smartroad: A crowd-sourced traffic regulator detection and identification system," Department of Computer Science, University of Illinois at Urbana-Champaign, Tech. Rep., 2013.

[24] X. Niu, Q. Zhang, Y. Li, Y. Cheng, and C. Shi, "Using inertial sensors of iPhone 4 for car navigation," in Proc. IEEE/ION Position, Location Navig. Symp., Myrtle Beach, SC, Apr. 2012, pp. 555-561.

[25] W. Feng, H. Zhao, Q. Zhao, and J. Li, "Integration of GPS and low cost INS for pedestrian navigation aided by building layout," Chinese J. of Aeronautics, vol. 26, no. 5, pp. 1283 - 1289, Oct. 2013.

[26] M. Fazeen, B. Gozick, R. Dantu, M. Bhukhiya, and M. Gonzalez, "Safe driving using mobile phones," IEEE Trans. Intell. Transport. Syst., vol. 13, no. 3, pp. 1462-1468, Sep. 2012.

[27] P. Händel, J. Ohlsson, M. Ohlsson, I. Skog, and E. Nygren, "Smartphone-based measurement systems for road vehicle traffic monitoring and usage-based insurance," IEEE Syst. J., vol. 8, no. 4, pp. 1238 1248, Dec. 2014.

[28] "10 reasons to unplug and unburden UBI," Driveway Software, 2014.

[29] J. Wahlström, I. Skog, and P. Händel, "Risk assessment of vehicle cornering events in GNSS data driven insurance telematics," in IEEE Conf. Intell. Transport. Syst., Qingdao, China, Oct. 2014, pp. 3132-3137.

[30] G. Castignani, T. Derrmann, R. Frank, and T. Engel, "Driver behavior profiling using smartphones: A low-cost platform for driver monitoring," IEEE Intell. Transport. Syst. Mag., vol. 7, no. 1, pp. 91-102, 2015.

[31] C. Saiprasert and W. Pattara-atikom, "Smartphone enabled dangerous driving report system," in 46th Int. Conf. Syst. Sci., Wailea, HI, Jan 2013, pp. 1231-1237.

[32] "Germany: Data protection autorities propose "the resolution" for the privacy of connected cars," Oct. 2014, Telematics Wire. 